\title{
The Application of Wavelet Transform in Real-time Data Compression of Power System
}

\author{
Jia $\mathrm{Wu}^{1, \mathrm{a}}$, Dan $\mathrm{Su}^{1}$, Wei Song ${ }^{1}$, Rui yang ${ }^{1}$, ShengPeng $\mathrm{Ji}^{2}$, Xianhui $\mathrm{Li}^{2, \mathrm{~b}}$, \\ Anmeng Wang ${ }^{3}$ \\ ${ }^{1}$ JiBei Electric Power Corporation, Beijing 100025, China; \\ ${ }^{2}$ China Realtime Database Co.,Ltd , Nanjing 210000, China. \\ aalice.0729@163.com, 'lixianhui@sgepri.sgcc.com.cn
}

Keywords: power system, Wavelet Transform, real-time data, data compression.

\begin{abstract}
For power system real-time data capacity is large, and the actual storage space is limited. This paper proposes a real-time data compression method based on wavelet transform. This method compress and decompress real-time data in power system through compactly supported orthonormal wavelet. The results show that this method achieves compression of the original data. It is possible to obtain a higher compression ratio and shorter compression time, and it is an effective real-time data compression method.
\end{abstract}

\section{Introduction}

With the development of automation and intelligent in power system, the real-time data is growing fast in electricity production. In order to improve production efficiency and optimize the operation, it must integrate a variety of real-time data. It is necessary to effectively save vast amounts of time series data formed many years, then power enterprise can use these data for analysis, mining and forecasting. The data in electricity production process is collected by various types of sensors, so it is dynamic. It is unrealistic to save the data in the form of raw data for long term, because the data collected by many sensors refresh fast, it is very large. So the study of data compression method suit for engineering applications and actual demand has become the urgent needs in power system[1-4].

Now the popular lossy compression algorithm are boxcar - backward slope method, revolving door compression algorithm and PLOT method[5-7]. Revolving door compression algorithm is more popular, it also knows as parallelogram algorithm. Overall, it is a linear trend compression algorithm, and its essence is to fit a series of consecutive data points by a determined straight line.

For specific data, this algorithm can find out the longest straight line trend as much as in a predetermined maximum allowable error range, that trend can be determined by the last two points, all points in the middle are discarded, so as to achieve the purpose of data compression [8].

Revolving door compression algorithm is relatively efficient in dealing with most real-time data channels, especially for the steady-state environment (without interference) can achieve very high compression ratio [9]. But the defect of this algorithm is also very obvious. Therefore, this article attempts to propose a compression method suitable for historical data which can efficiently compress steady and unsteady historical data and also can fast storage and access this data[10].

This paper proposes a time series data compression method based on wavelet transform. First, it processes time series data through wavelet decomposition technology, then abandons wavelet coefficients which have smaller mold through setting the threshold value, and then designs a compact compression to storage data after conversion.

\section{Wavelet Transform Algorithm}

Define $\Psi(t) \in(R)\left(L^{2}(R)\right.$ as square integrable real space, namely it is finite energy signal space, its Fourier transform ${ }^{\stackrel{\leftrightarrow}{(}(\omega)}$, when ${ }^{\hat{\Psi}(\omega)}$ meet permit conditions, 


$$
C_{\Psi}=\int_{R} \frac{|\stackrel{\Delta}{\mid \Psi(\omega)}|^{2}}{|\omega|} d \omega<\infty
$$

Define $\psi(\mathrm{t})$ as a basic wavelet or mother wavelet. Scale and translate the generating function $\psi(\mathrm{t})$, it can get a wavelet sequence. For continuous case, wavelet sequence is:

$$
\Psi_{a, b}(t)=\frac{1}{\sqrt{|a|}} \Psi\left(\frac{t-b}{a}\right) \quad a, b \in R ; \quad a \neq 0
$$

Wherein a is a stretch factor, $\mathrm{b}$ is the translation factor.

In the case of discrete, wavelet sequence is:

$$
\Psi_{j . k}(t)=a_{0}^{-j / 2} \Psi\left(\frac{t-k a_{0}^{j} b_{0}}{a_{0}^{j}}\right)=a_{0}^{-j / 2} \Psi\left(a_{0}^{-j} t-k b_{0}\right) \quad j, k \in z
$$

Binary wavelet is used commonly. Binary Wavelet signal has a zoon effect for signal analysis. Assuming there is a magnification 2-j, and it corresponds to a certain part of the observed signal. If want to watch smaller details of the signal, can increase the magnification the same as decreases the value of $\mathrm{j}$. conversely, if want to understand the content of signal more coarse, increase $\mathrm{j}$ value.

\section{Time Series Data Compression Based on Wavelet Transform}

The time series data compression method based on wavelet transform reserves wavelet approximation coefficients, then retains the wavelet detail coefficients with a larger modulus value through setting the threshold, and discards the detail coefficients with smaller modulus value corresponding the noise and redundant information, in order to achieve the original data compression.

Specific compression process is as follows:

Collect real-time data, save data into the historical data buffer queue according to the time order.

Determine buffer data: setting a limit value of the amount of data to be processed, compare the amount of historical data obtained from the history buffer queue with the limits, if exceed the limit, then convert the historical data by wavelet transform, otherwise, continue to obtain historical data from the historical data buffer queue.

Wavelet transform: convert reorganization series data by Daubechies 10 wavelet transform, Daubechies is compactly supported cross wavelet, has a good effect.

Threshold treatment: use pre-set threshold, if detail coefficient is less than the threshold value after wavelet transform then set it to 0 ,

If the details of the wavelet transform coefficients of the coefficient is less than the threshold value is set to 0 , as follows:

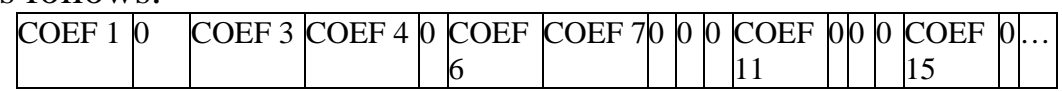

Data storage: the data processed by the above-mentioned threshold is jointly represented by the flag and the data bits, and stored in the file system, as follows:

Flag

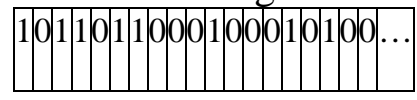

Data bits

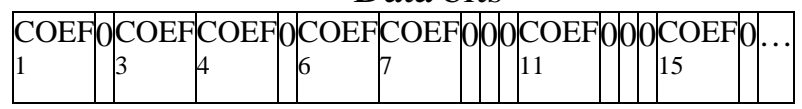

Because determine whether a data is 0 simply needs a bit, so the number of time-series data for each wavelet transform is an integer multiple of 8 . As time series data in power system changes more laws, accordingly detail coefficients are relatively close to zero. So ideally a 4 bytes of raw data just use 1 bit to save, the data compression rate can be accessible 32: 1 .

Data decompression: Reads the flag bits and data bits from the file system, and read the details from the data bits and fill factor according to the position 1 in flag, form a group detail coefficients as shown below:

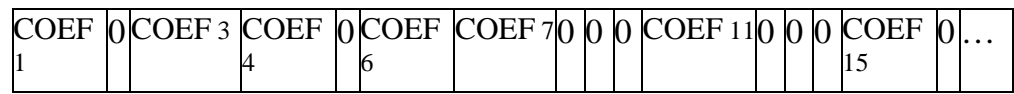

Then process data through wavelet transform according to this group of coefficients, and it can get the data after compression.

This compression method belongs lossy compression algorithm, because there is noise in the data 
acquisition system itself, the substance of wavelet compression and wavelet denoising is same, so for processing applications in the compressed data, these compression errors do not have a big impact. Or in a sense, this algorithm can remove the noise effectively, and make data to be closer to the original data.

\section{Experimental results}

The following are some examples of power system measurement real-time data for compression which adopting Daubechies 10 wavelet method. the data quantity is about 1024 .

With the wavelet transformation, it get the detailed coefficient as below:

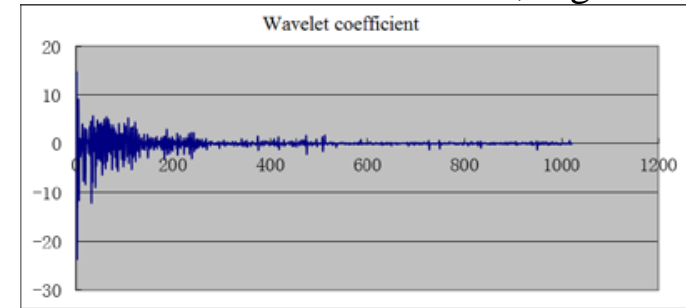

Fig.1 the wavelet coefficient of Daubechies 10 wavelet

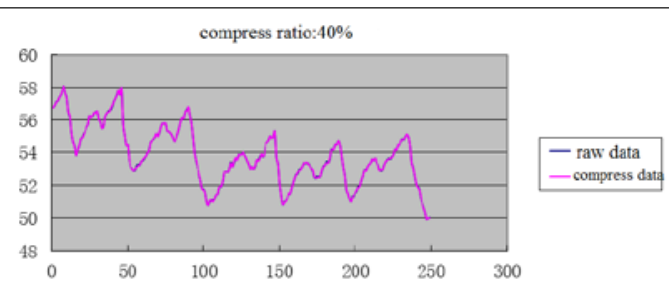

Fig.2 the compress ratio with the data error is 0.001

From the diagram, the most coefficients is 0 or close to 0 , if the accuracy of the data error is 0.001 , then the compress ratio is 0.4 .

If the accuracy of the data error is 0.01 , then the compress ratio is 0.95 . As show Fig3.

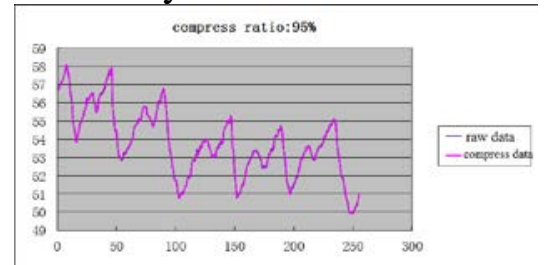

Fig. 3 the compress ratio with the data error is 0.01

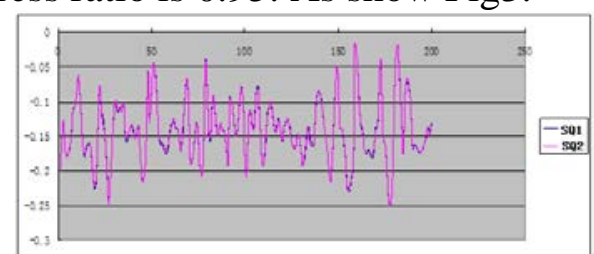

Fig. 4 the compress ratio of jitter data with the data error is 0.001

If we do the experiment with jitter data, With the same accuracy of data error of 0.01 , the compress ratio of irregular data is lower than the regular one whose compress ratio is 0.26. As show Fig.4.

If we do the experiment with a group of more regular data, we can see that with the accuracy of the data error of 0.0001 , the compress ratio is 0.9 ,which is also very high. Show as Fig.5.

With the above data, if we make the comparison between wavelet transformation(WT) and Swing Door(SW) compressing in real-time data compress, we can get that the performance of compression is close. both of them are in the tolerance. The result is show as Fig.6.

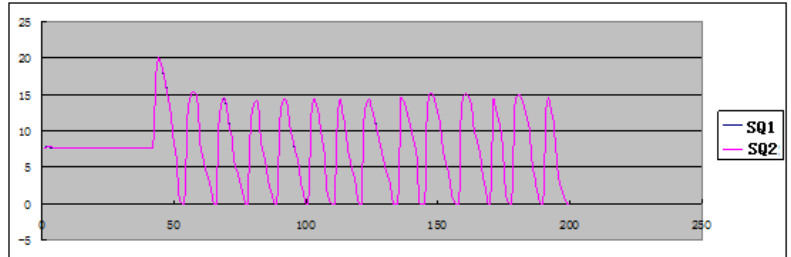

Fig. 5 the compress ratio of regular data with the data error is 0.0001

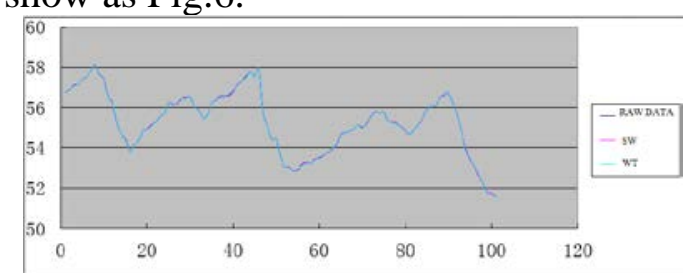

Fig.6 The comparison between wavelet transformation(WT) and Swing Door(SW)

On the other hand, we applied the test of timeliness to wavelet transformation compressing, we can get the wavelet response to regular or irregular tests according to the timeline show as Fig.7.

The relationship between data quantity and time of irregular exchange data show as Fig.8.

The diagram above shows that with the data quantity between 56 and 16384, the consumption time of compressing or uncompressing is proportional to the quantity of data. Because we need to consider the appropriate tolerance, it will take more time of uncompressing than compressing, so no matter compress or uncompressing. The unit of time is millisecond. 


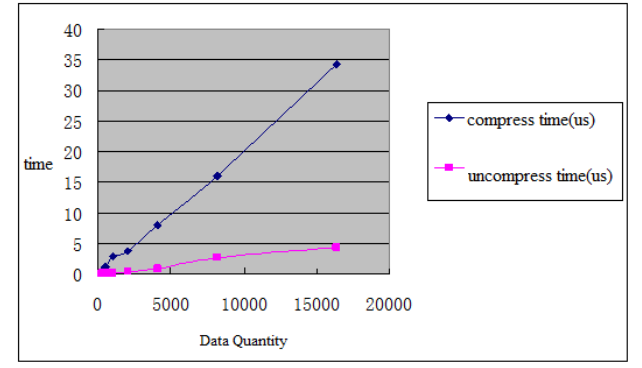

Fig.7 The relationship between data quantity and time consumption of regular exchange data

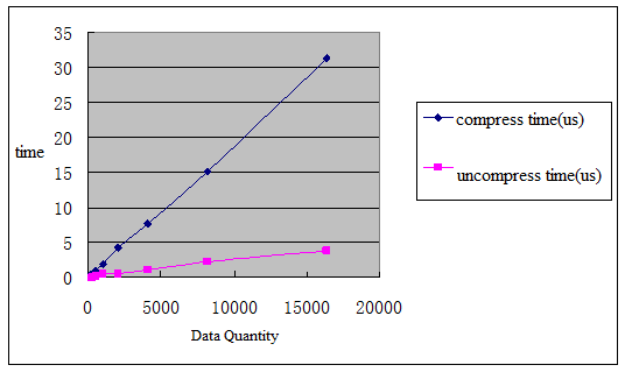

Fig.8 relationship between data quantity and time consumption of irregular exchange data

\section{Conclusion}

This paper proposed a power system real-time data compress method based on wavelet transformation. It verified by read data. With the result shows that this method is effective and correct. It is Wide application value in power system real-time data compress, because the compressing ratio is high, the loss of data is low and with the effect of noise reduction in raw data. Also, wavelet transformation data compress time consuming is small, when the value is smaller than 10000 points, the unit of time consuming is kept about millisecond, which is totally satisfied in the real-time processing of power system. This method can save the space for storing real-time data, and it can ensure the performance of data uncompressing. so it is convenient in the application of project, it can promote the intelligent power system to achieve a new era.

\section{References}

[1]SUN Peixia, GUO Qing, WANG Qian, et al. Two-dimensional discrete wavelet transform compression algorithm for power quality data [J]. Journal of Computer Applications, 2014, 34(A01): $234-237$.

[2] MA Fayong, LI Qipeng, MA Zhibin, et al. The Research of Historical Data Compression and Storage Strategy in Power Dispatch SCADA System [J]. Power System Technology, 2014, 38(4): 1109 - 1114.

[3] ZHANG Chong -zlAppdication of PI database in power grid informationization [J]. Modern Electronics Technique, 2014, 37(4): 76 - 77.

[4] YANG Yong-jun, XU Jiang, SHU Yi, et al. Research on Lossless Data Compression Algorithm for Historical Data of Real-time Database [J]. Computer and Modernization, 2012, 0(6): $165-168$.

[5] Liu Hui, Zhang Yun, Zhang Xiangjing, et al. Application of PI Real-time Data Management System [J]. Automation in Petro-Chemical Industry, 2013, 49(1): 47 - 49.

[6] Xiao Jianrong, Wan Hui. Application of Aspen InfoPlus.21 Real-time Database Management System [J]. Automation \& Instrumentation, 2013, 0(3): 120 - 121.

[7] XING Rui, QI Qi, ZHENG Tao. Improved SDT algorithm [J]. Computer Engineering and Design, 2013, 34(2): 515 - 518.

[8] FU Yuan-di, ZHANG Yan-yuan, LIN Yi. Data Acquisition and Storage System Based on Online Compression [J]. Computer and Modernization, 2014, 0(7): 49 - 52.

[9] Yu Songtao, Wang Xiaokun, Zhao Liqiang, et al. Improved Algorithm of Revolving Door (SDT) Based On Tolerance Dynamically Adjusted [J]. Journal of Beijing University of Chemical Technology, 2013, 40(3): 109 - 113.

[10] LING Xiao-ming, HAO Yu-sheng • Research on a Disk History Database Model [J]. Computer Engineering, 2014, 40(5): 26 - 30. 